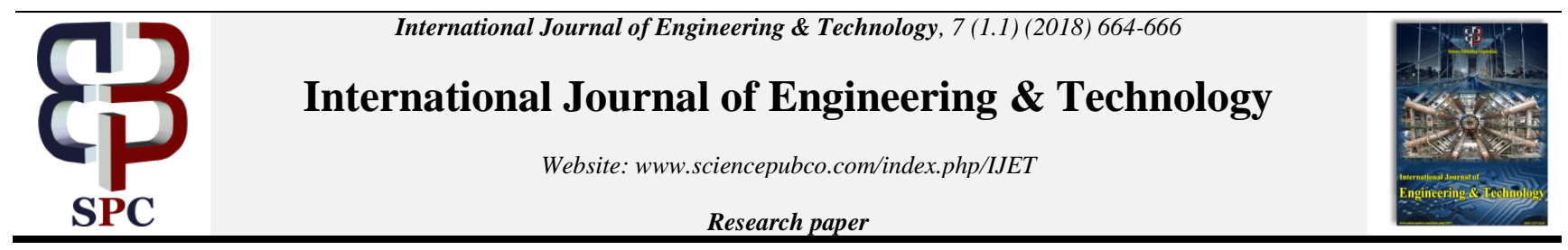

\title{
An efficient and secured approach for sequential and transparent identity validation of user in internet services
}

\author{
P. Kalyan Chakravathy ${ }^{1 *}$, K. Vasavi Devi ${ }^{2}$, T. Sai Sri ${ }^{2}$, Sk. Abu Saleha ${ }^{2}$ \\ ${ }^{1}$ Asst. Prof, Department of computer science and Engineering, Koneru Lakshmaiah Education Foundation, Andhra Pradesh, India \\ ${ }_{2} 4^{\text {th }}$ B. Tech, Department of computer science and Engineering, Koneru Lakshmaiah Education Foundation, Andhra Pradesh,India \\ *Corresponding author E-mail: kluchakri@gmail.com
}

\begin{abstract}
These days, it ends up noticeably genuine worry to give greater security to web administrations. Along these lines, secure client verification is the central assignment in security frameworks. Generally, a large portion of the frameworks depend on sets of username and secret key which checks the personality of client just at authentication stage. Once the client accesses with username and secret key, no checks are performed and encourages amid working sessions. Be that as it may, rising biometric arrangements gives the username and secret key using biometric information of client. In such approach, single shot check is less proficient in light of the fact that personality of client is perpetually amid entire session. Consequently, an important arrangement is to utilize brief time of timeouts for every session and occasionally ask the client to enter his or her qualifications again and again. In any case, this isn't a legitimate arrangement since it vigorously influences the administration convenience and eventually the fulfilment of clients. This paper investigates the framework for nonstop verification of client utilizing his accreditations, for example, biometric qualities. The utilization of consistent biometric verification framework procures certifications without expressly telling the client or requiring client communication that is, straightforwardly which is essential to ensure for better execution and administration ease of use.
\end{abstract}

Keywords: Authentication; Biometric Authentication; Continuous User Verification; Web Security.

\section{Introduction}

In relatively every part of human life have registering gadgets, (for example, PC, advanced mobile phone, tablet, or shrewd watches) end up noticeably critical devices. The correspondence administrations, flying and money related administrations are particularly controlled by PC frameworks. Individuals endow with indispensable data, for example, restorative and criminal records, oversee exchanges, pay bills and private reports. Notwithstanding, this expanding reliance on PC frameworks, combined with a developing accentuation on worldwide openness in the internet, has disclosed new dangers to PC framework security. Likewise, violations and shams in the internet are all over the place. For most existing PC frameworks, once the client's personality is checked at login, the framework assets are accessible to client until he/she exits the framework or locks the session[1]. Actually, the framework assets are accessible to every client who amides that period. This might cause for low security situations, yet can prompt for session seizing, in which assailants focuses on an open session, e.g. at the point when each individuals leaves the PC not accessed for shorter or longer periods when it is opened, for instance to get some espresso, to go and converse with a partner, or just on the grounds that they don't have the propensity for locking a PC on account of the bother. In most hazardous situations or where the cost of unapproved utilization of PC is high, a ceaseless access of the client's personality is critical. By utilizing nonstop check the personality of the human working the PC is persistently confirmed. Username and secret word of conventional verification framework is get supplant by biometric quality in the event of biometric method. Biometrics are the science and innovation of deciding and distinguishing the right client personality in light of physiological and behavioural characteristics which incorporates confront acknowledgment, retinal outputs, unique mark voice acknowledgment and keystroke elements. Biometric client confirmation is figured as a solitary shot check .Single shot confirmation gives client confirmation just at the login time. On the off chance that the personality of client is confirmed once, at that point assets of the framework are accessible to client for settled timeframe[2] and the character of client is lasting for entire session. An essential or important arrangement is to maintain the use of short session timeouts or occasionally ask for the client to include his/her qualifications over and over. To conveniently distinguish abuses of PC assets and keep that an unapproved client and replaces the approved one and proposed arrangements in light of multi-modular bio-metric consistent validation, transforming client confirmation into a persistent process instead of one-time event. To stay away from that, a solitary biometric attribute is designed and biometrics validation depends on different biometrics qualities and new approach for session administration and client's confirmation are talked in this paper is characterized and actualized with regards to the multi-modular biometric verification framework which is known as (CASHMA) Context Aware Security by Hierarchical Multilevel Architecture. The CASHMA framework understands the benefits of the protected biometric validation on the Internet, in this clients just need to recall a single username or id and by using their biometric information instead of passwords to verify in various web administrations. CASHMA 
work safely with any sort of web benefit for instance web based managing an account, military zones, and air terminal zone which require high security administrations [3-7]

\section{Literature survey}

The prologue to the security problems and its worry is portrayed in earlier area. In this paper, we have contemplated before investigate papers and identified the ordinary confirmation frameworks which presents the single time verifications of the client. The classifications of the authentication and security frameworks rely upon quality of assault and they are grouped into solid and feeble. The compressing investigations of prior research is as per the following:

1) The Primary approach is the information based character for verification of client includes is watchword id is the thing which you know and password contains single word, Personal Identification Number otherwise known as PIN, Phrases which can be saved mystery for confirmation. Yet, this approach which is Knowledge based personality does not offer any great arrangement, it can be looked or figured out by an aggressor and security against revocation [6] is not presented.

2) The Secondary approach is based on the character which is known as question based for verification or authentication of client which includes what you have is token; Token means a physical gadget which gives confirmation that tokens can be secured, get to token, stockpiling gadgets including the passwords, for example, bank cards [6] or brilliant card. The primary weakness is that the Identity token can be lost or stolen which bother and cost.

3) The Third most or the last approach is ID based confirmation for validation of client it considers your identity, which is just biometric. For example, figure print ID, confront acknowledgment, voice acknowledgment and mark or eye examine give more grounded resistance against assault Knowledge based Contrasting and protest/element based ID based confirmation gives advantaged in the security level.

\section{The cashma architecture}

The CASHMA architecture using Hierarchical Multilevel Architectures gives Context-Aware Security. This framework is used for securing biometric validation on the web. CASHMA works safely with any sorts of web benefits and in incorporating administrations with high level of security requests as web based by keeping money administrations. Contingent upon necessities of the proprietor of the web benefit the CASHMA confirmation benefit supplant the customary validation benefit and the preferences.

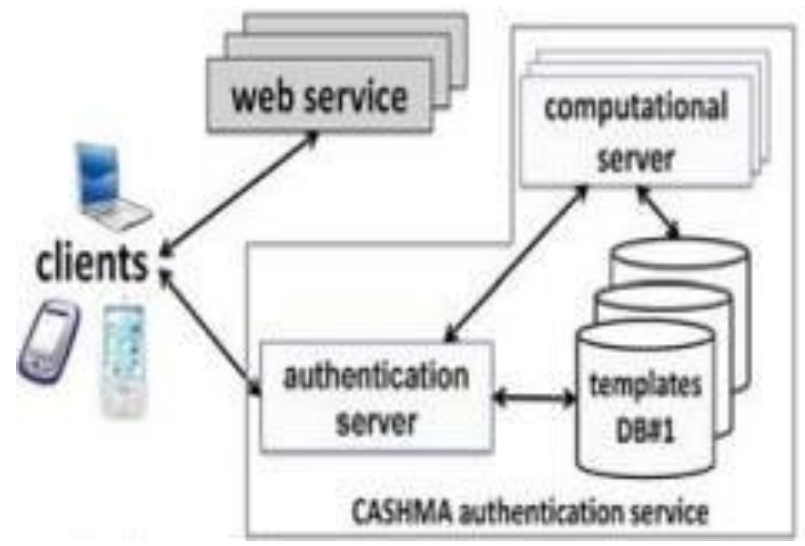

Fig. 1: Overall View of CASHMA Architecture.

The framework engineering is comprising of the benefits of the CASHMA confirmation, the web administrations and the customers, they are associated through correspondence channels. Fig. 1 shows the consistent verification framework to the web benefits. The validation server, which collaborates with the computational servers, customers who performs correlations of biometric information for check of the clients, and databases of formats which contains the biometric layouts of the clients (that are required for client confirmation or check reason). The web benefit requests the verification of clients to the confirmation server CASHMA. These administrations are any sort of Internet benefit. At long last, we mean the clients' gadgets like (PCs, Desktop PCs, tablets, and so forth.) by customers which secure the biometric information relating to the clients of different biometric characteristics, and transmit those information to the CASHMA verification server towards an objective web benefit. A customer contains. I) the CASHMA application - transmits the crude information to the confirmation server, ii) Sensors - secure the crude information. The CASHMA confirmation uses server to apply client validation and check systems that contrast the crude information and puts away the biometric formats. Consider web based managing an account where a client needs to sign into a web based saving money benefit utilizing an advanced cell. Here web administrations and client must be selected to CASHMA verification administration and client on his advanced cell introduced CASHMA application [4]. The cell phone contacts the web based keeping money benefit, which answers to get a confirmation authentication by asking the customer to contact the CASHMA verification server. Utilizing the CASHMA application, the mobile phone sends its remarkable identification and biometric information to the validation server for check. The confirmation server checks the client character, and allows the entrance on the off chance that: I) benefit it has rights to get to the web based managing an account administration, ii) it is enlisted in the CASHMA validation[5] and, iii) the obtained biometric information coordinate those put away in the formats database related to the identifier. If there should be an occurrence of effective client check, to the customer the CASHMA validation server discharges a verification endorsement, demonstrating its personality to outsiders, and incorporates a timeout of the client session which sets the greatest span. The customer displays this endorsement to the web benefit, which confirms it and gifts access to the customer. For keeping up the session open the CASHMA application works constantly: it straightforwardly procures biometric information from the client, and to get another authentication it sends them to the CASHMA confirmation server. Such testament, is sent to the web administration to additionally expand the client session to

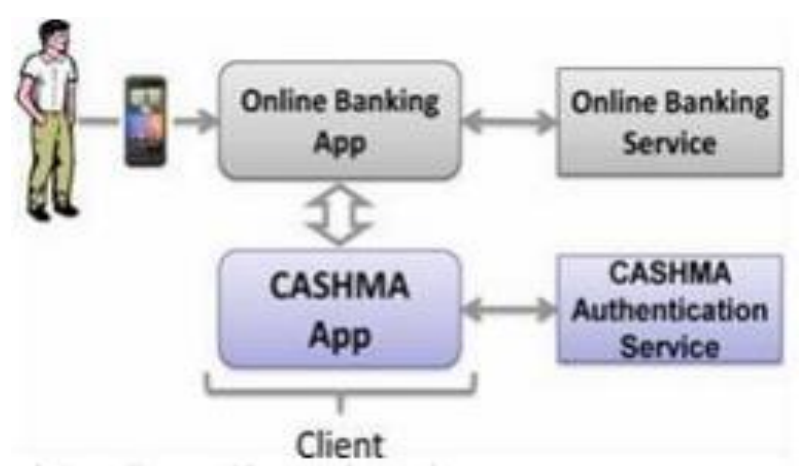

Fig. 2: Online Banking Services Using CASHMA Incorporates Another Timeout.

\section{The cashma certificate}

The data which is contained in the body of the CASHMA testament [6] by utilizing the CASHMA confirmation server is transmitted to the client, basic to perceive critical purposes of the convention. The CASHMA declarations comprise of grouping number univocally and Time stamp distinguish each endorsement, and it care for from replay assaults. Id e.g., a number, is the individual id. Decision speaks to the last consequence of the confirmation procedure which is done on the server side. By the CASHMA 
verification server it incorporates the sessions lapse time, progressively allotted. Commonly, the worldwide trust organize and the session timeout are consistently registered by method for considering the time prompt in which the CASHMA application procures the biometric information, to limit potential issues concerning obscure deferrals in discussion and calculation. Because of the reality such deferrals won't be predicable in earlier, essentially providing a relative timeout incentive to the client won't be suitable, so the CASHMA server in this manner gives unquestionably the prompt of time at which the session must terminate. The CASHMA authentications will most likely be lapsed when the termination timeout accomplish zero.

\section{The continuous authentication protocol}

The ceaseless confirmation convention permits giving versatile session timeouts to a web administration with a customer to set up and keep up a protected session. The timeout is adjusted based on assume, that the CASHMA validation framework puts in the client and in the biometric subsystems. The convention execution is of two successive stages which is the underlying stage and the support stage. In the underlying stage, we intend to verify the client into the framework and with the web benefit we build up the session. Amid the support stage, when client personality confirmation is performed utilizing new crude information gave by the customer to the CASHMA validation server, the session timeout is adaptively refreshed. The client (the customer) contacts the web benefit for an administration ask for, the web benefit answers that a legitimate declaration from the CASHMA confirmation benefit is required for verification[7-9].

\section{Conclusion}

Session administration framework is completely in view of username and sessions, and secret key are ended by the lapse of session timeouts or unequivocal logouts. Techniques utilized for consistent validation utilizing distinctive biometrics. Beginning the one time login confirmation which is associated with post signed in session is lacking to address the hazard. We misused the novel plausibility acquainted by biometrics with characterize a convention for constant confirmation that enhances security and ease of use of client session. The convention processes versatile timeouts based on the confide in postured in the client action and in the quality and sort of biometric information procured straightforwardly through checking in foundation the client's activities. Constant confirmation check with convenience of client session and multi - modular biometrics enhances security. The capacities which are proposed for the assessment of session timeout are chosen among an expansive arrangement of conceivable options.

\section{References}

[1] CASHMA-Context Aware Security by Hierarchical Multilevel Architectures, MIUR FIRB, 2005.

[2] Andrea Ceccarelli, Leonardo Montecchi, Francesco Brancati, Paolo Lollini, Angelo Marguglio, Andrea Bondavalli "Continuous and Transparent User Identity Verification for Secure Internet Services" IEEE Transaction on De-pendable and Secure Computing, VOL 12,NO. 3, JUNE 2015. http://www.ijtra.com/view/continuous-andtransparent-user-identity-verification-for-secure-internetservices.pdf

[3] Anil K. Jain, SharathPankanti, SalilPrabhakar, Lin Hong, Arun Ross, James L. Wayman,"Biometrics: A Grand Challenge" International Conference on Pattern Recognition, Aug 2004. http://biometrics.cse.msu.edu/Publications/GeneralBiometrics/Jaine tal_BiometricsGrandChallenge_ICPR04.pdf

[4] T. Sim, S. Zhang, R. Janakiraman, and S. Kumar, "Continuous Verification Using Multimodal Biometrics," IEEE Trans. Pattern Analysis and Machine Intelligence, vol. 29, no. 4, pp. 687-700, Apr. 2007. https://pdfs.semanticscholar.org/b6eb/44fbd7de0a7637b0aaccf0bb4 62c12d19e67.pdf

[5] T.F. Dapp, "Growing Need for Security in Online Banking: Biometrics Enjoy Remarkable Degree of Acceptance,"” Banking \& Technology Snapshot, DB Research, Feb. 2012 http://www.ijrter.com/papers/volume-2/issue-8/continuous-userauthentication-using-cashma-system.pdf

[6] A. Ceccarelli, A. Bondavalli, F. Brancati, and E. La Mattina, "Improving Security of Internet Services through Continuous and Transparent User Identity Verification," Proc. Int'l Symp. Reliable Distributed Systems (SRDS), pp. 201-206, Oct. 2012.

[7] L. Montecchi, P. Lollini, A. Bondavalli, and E. La Mattina, "Quantitative Security Evaluation of a Multi-Biometric Authentication System," Proc. Int'l Conf. Computer Safety, Reliability and Security, pp. 209-221, 2012.

[8] S. Kumar, T. Sim, R. Janakiraman, and S. Zhang, "Using Continuous Biometric Verification to Protect Interactive Login Sessions," Proc. 21st Ann. Computer Security Applications Conf. (ACSAC '05), pp. 441-450, 2005. http://ijarcsse.com/Before_August_2017/docs/papers/Volume_6/9_ September2016/V619-0141.pdf.

[9] Biometric System Base Secure Authentication Service for Session Management NilimaDeore, Prof.C.R.Barde International Journal of Innovative Research in Computer and Communication Engineering Vol. 3, Issue 12, December 2015. 